\title{
METEOROID PROPERTIES FROM PHOTOGRAPHIC RECORDS OF METEORS AND FIREBALLS
}

\author{
ZDENĚK CEPLECHA \\ Academy of Sciences of the Czech Republic, 25165 Ondřejov Observatory
}

\begin{abstract}
Statistical criteria based on the single-body theory enabled the distinction of different composition groups of meteoroids in the past. The new single-body model proposed by Pecina and Ceplecha $(1983,1984)$ is capable of determining the individual values of ablation coefficients, which has proved to be a better tool for separating meteoroids of different ablation properties. However, a significant fraction of fireballs exhibit a time-dependence of residuals, when the single-body model is applied to their photographic observations. This was recently explained by assuming sudden fragmentation at a point (gross-fragmentation). The proposed gross-fragmentation model was checked in exceptional cases, when splitting of a fireball was directly visible on the photographs. The new fragmentation model was then applied to the best photographic records of Prairie Network fireballs (PN). Least-squares fit of computed to observed distances along a meteoroid trajectory determines uniquely the ablation coefficient, the shape-density coefficient, the position of the gross-fragmentation point and the amount of fragmented material relative to the main body mass. This enabled not only a better classification according to ablation coefficient (composition groups), but also a recognition of different strength categories according to dynamic pressure at the fragmentation point. Except for composition groups (types) I, II, IIIA, IIIB, each meteoroid with precise photographic data on its fireball can be classified as NF (no-fragmenting), 1F (with one point of fragmentation) and MF (with many points of fragmentation). The fragmenting meteoroids ( $1 \mathrm{~F}$ and $\mathrm{MF}$ ) can moreover be sorted into several categories $(a, b, c, d, e)$ according to dynamic pressure at the fragmentation point. Thus the classification became two dimensional, separating meteoroid composition from structure. Values of ablation coefficients and bulk-densities were revised using this model. The amount of fragmented mass relatively to the main body was also determined. Typical sudden fragmentation for almost half of all fragmenting meteoroids is equivalent to stripping away slightly more than half of the mass.
\end{abstract}

\section{Classification from preheating and ablation.}

When a meteoroid collides with the Earth's atmosphere, most of the mutual kinetic energy is freed in interaction processes. The meteor phenomenon is thus very much dependent on the composition and structure of the meteoroid. Even the preheating is a sensitive indicator of the physical properties of the material from which the meteor body is composed.

The beginning heights of the luminous trajectories can thus be used for classification of meteoroids (Ceplecha 1967, 1968). This was done in the past for bodies in the mass range from $2 \times 10^{-8} \mathrm{~kg}$ to $0.5 \mathrm{~kg}$ by recognizing different discrete levels of beginning heights. Four groups denoted A, B, C, and D can be specified. The A group meteors start their luminous trajectory much lower down than the $D$ group meteors. The ratio of the air density at the beginning height between the two most populated groups $A$ and $C$ is a factor of 5 , which corresponds to a difference in the normalized beginning heights of $11 \mathrm{~km}$ between $A$ and $C$ groups. Almost the same 
difference is obtained between $\mathrm{C}$ and $\mathrm{D}$ group, thus the total difference between $A$ and $D$ group is $21 \mathrm{~km}$ reflecting the difference of the material constants (heat conductivity, heat capacity, bulk-density and radiation emissivity). The levels are schematically presented in Fig. 1. Details on all previous work are summarized in Ceplecha (1988).

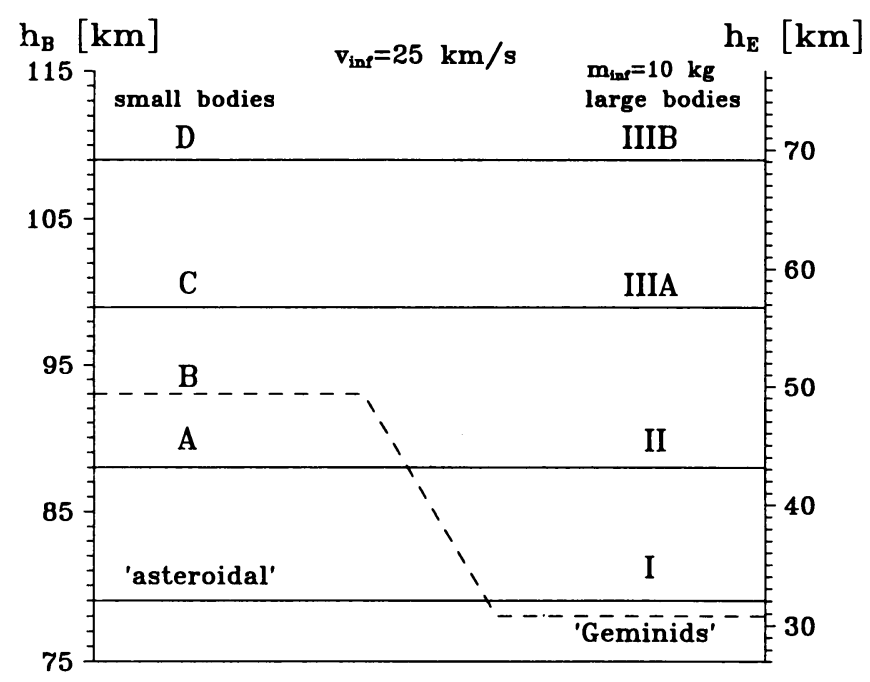

Fig. 1. Schematic representation of discrete levels of meteor beginning heights, $h_{B}$, and of meteor terminal heights, $h_{E}$, and their mutual relationship.

Bodies larger than $0.1 \mathrm{~kg}$ can be better classified according to their ability to penetrate the atmosphere. These bodies produce fireballs and our knowledge of them comes from photographic observations of fireball networks (EN, PN, MORP; Ceplecha, 1988; Ceplecha and McCrosky, 1969; McCrosky et al., 1971; Halliday et al., 1984, 1989). Except for very early portions, the ablation part of their trajectories is practically identical with their luminous trajectories. Thus terminal heights reflect the ablation ability and are an excellent indicator of the composition and structure of these larger bodies (Ceplecha and McCrosky, 1976). Terminal heights have to be normalized to the same velocity, to the same mass and to the same inclination of the trajectory to the horizon, when we want to use them for classification of fireball bodies. These normalized terminal heights for large meteor bodies cover a huge range of $1: 1000$, if expressed as ratios of the air densities at the terminal point.

The fireball networks collected data on meteoroids in the mass range from $0.1 \mathrm{~kg}$ to $5 \times 10^{4} \mathrm{~kg}$; the upper limit corresponds to a size of $7 \mathrm{~m}$. In this mass range we can again recognize several discrete levels of these normalized terminal heights : members of these levels were denoted as group I, II, IIIA and IIIB. The terminal height levels for fireballs are schematically related to the beginning height levels for 
TABLE I

Survey of populations of large meteoroids ( $\rho=$ bulk density, $\sigma=$ ablation coefficient).

\begin{tabular}{|c|c|c|c|c|c|c|}
\hline \multirow{2}{*}{$\begin{array}{c}\text { population } \\
\text { (type) }\end{array}$} & \multirow{2}{*}{$\stackrel{\rho}{\mathrm{g} / \mathrm{cm}^{3}}$} & \multirow{2}{*}{$\begin{array}{c}\sigma \\
\mathrm{s}^{2} / \mathrm{km}^{2} \\
\end{array}$} & \multicolumn{3}{|c|}{$\begin{array}{c}\text { characteristic orbit } \\
\text { for } 0.1-1 \mathrm{~m} \text { size }\end{array}$} & \multirow[t]{2}{*}{$\begin{array}{l}\text { assumed } \\
\text { composition }\end{array}$} \\
\hline & & & $a$ & $e$ & $i$ & \\
\hline I & 3.7 & .017 & 2.4 & .68 & $6^{\circ}$ & stony \\
\hline II & 2.0 & .041 & 2.3 & .61 & $5^{\circ}$ & carbonaceous \\
\hline IIIA & 0.75 & .10 & 2.4 & .82 & $4^{\circ}$ & cometary \\
\hline IIIAi & 0.75 & .10 & $\approx \infty$ & .99 & random & cometary \\
\hline IIIA[C3] & 0.75 & .10 & 2.7 & .67 & random & cometary \\
\hline IIIB & 0.27 & .21 & 3.0 & .70 & $13^{\circ}$ & soft cometary \\
\hline
\end{tabular}

faint photographic meteors in Fig. 1. A summary of all the fireball groups with data on average ablation coefficients and bulk densities are given in Table I. Enormous variety of compositions and structures exceeding our "surface experience" from studying meteorites is evident : meteorites are just samples of the strongest part of interplanetary matter coming to the Earth, which survived the atmospheric entry (groups I and II). The group I level belongs to stony meteorite falls as proved by Príbram, Lost City and Innisfree meteorite falls. The asteroidal origin of these bodies 'is evident. Group II is suspected to be composed of several systems of carbonaceous bodies of both asteroidal and cometary origin. The presence of carbon in spectra of meteoroids of this group is one of several verifications of the proposed composition. Group IIIA and IIIB are groups with meteoroids of cometary origin as it is evident from individual coincidences with meteor streams. The low density cometary material similar to the meteoroids from P/Giacobini-Zinner belongs to group IIIB. Recent statistical studies of bodies in the mass range from $10^{4}$ to $10^{7} \mathrm{~kg}$ (sizes of the order of $10 \mathrm{~m}$ ) based partly on extrapolations of fireball data (Ceplecha, 1988) and partly on Spacewatch telescope discoveries of very small "asteroidal" bodies (Rabinowitz, 1993), yielded evidence that most of these bodies belong to IIIB group (Ceplecha, 1992). Thus the $10 \mathrm{~m}$ size bodies in the solar system may be mostly inactive material of cometary composition and structure. Alternatively, they may also belong to some not yet known system of bodies, which is relatively very strong at $10 \mathrm{~m}$ sizes.

Statistical criteria and studies based on the single body theory enabled distinction of all these different composition groups in the past (Ceplecha and McCrosky, 1976; Ceplecha and Borovička, 1992; Sekanina, 1983; Wetherill and ReVelle, $1981 \mathrm{a}, \mathrm{b})$. The biggest disadvantage of statistical procedures was the impossibility of separating the value of ablation coefficient from the bulk density of meteoroids : this could only be done in a statistical way (Table I contains these "statistical" values). 


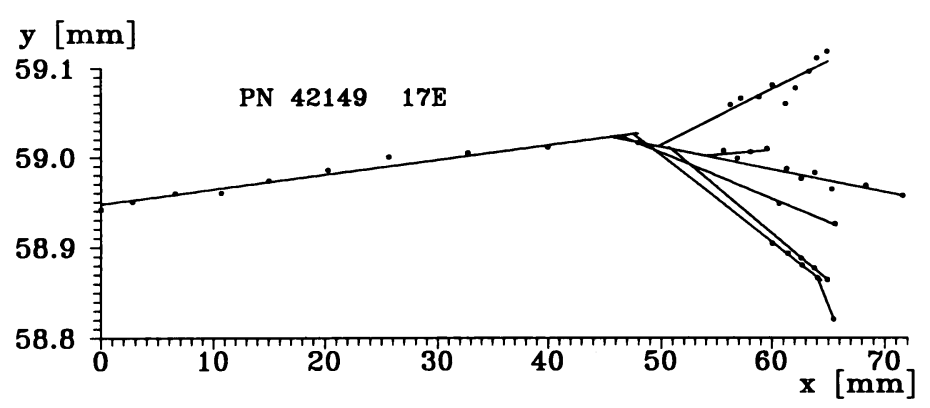

Fig. 2. Splitting trails of fireball PN 42149. The $x$ and $y$ are rectangular coordinates defined by the measuring device (Ascorecord). The individual measured points of different splitting trails are given as dots. Least-squares fits of straight lines to these points define trails of individual fragments. The $y$-axis is greatly enlarged to better visualize the separation of individual fragments. The main trail is terminated at the largest $x$-value.

\section{Gross fragmentation}

A new single body model was proposed by Pecina and Ceplecha $(1983,1984)$. This model was applied to individual well-observed meteoroids (multi-station photographs) and yielded the possibility of separating the ablation coefficient from the bulk density. This method was recently used to all available data on PN and EN fireballs with the result of better individual values of ablation coefficients (Ceplecha and Borovička, 1992). At the same time a new phenomenon was revealed : grossfragmentation, i.e. a sudden instantaneous fragmentation (Ceplecha and McCrosky, 1992). When the time dependencies of residuals received from single body solutions were inspected by simple graphical means, the gross-fragmentation seemed to be important for about $25 \%$ of all observed fireballs.

Recently a new model of gross-fragmentation was proposed and checked on by means of several cases, where the fragmentation is directly visible as splitting trails on the photographs (Fig. 2). The gross-fragmentation model gives the distance flown by the meteoroid along its trajectory, $l$, as a function of time : $l=l(t)$. This distance is also directly derived as $l_{o b s}$ from measured values on the multistation meteor photographs, i.e. from apparent distances along the trail measured for each time mark (velocity is an indirectly derived value and moreover it is the first derivative of the measured distances). The mathematics belonging to this model, the procedures used, and many details and results of application to PN fireballs, can be found in Ceplecha et al. (1993). Together with the observed heights, $h_{\text {obs }}$, which are available for each time mark, $t$, the observed distances, $l_{\text {obs }}$, determine uniquely the ablation coefficient, the shape-density coefficient, the position of the gross-fragmentation point, and the amount of fragmented material relatively to the main body mass just by means of the least squares fit of $l$ to $l_{\text {obs }}$. (Fig. 3).

A computer program for automatic searching for gross-fragmentation points was prepared and applied to a sample of 80 records of PN fireballs with precise values of the measured distances and heights. From those 21 proved to be without gross- 


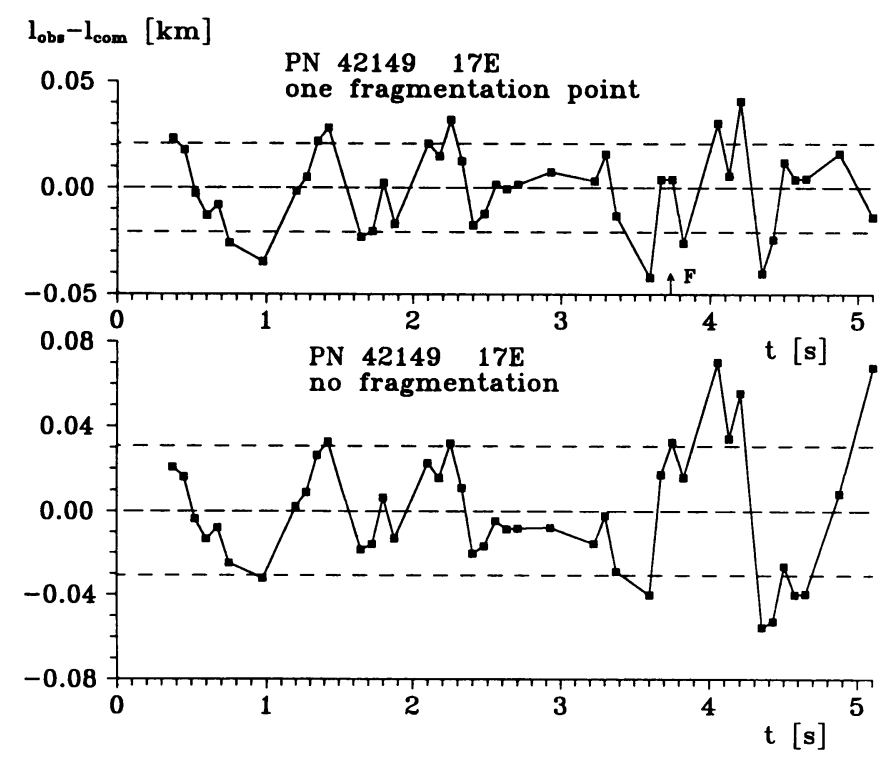

Fig. 3. Residuals, $l_{o b s}-l_{\text {com }}$, of solutions without fragmentation and with one fragmentation point. The position of the fragmentation point (denoted by F) is derived from the least squares solution and can be compared with the geometrically determined fragmentation point (see Fig. 2). Residuals for the one-fragmentation-point solution are smaller and almost time-independent.

fragmentation (Table IV : class NF), 19 having one point with an overwhelming amount of fragmentation (class $1 F$ ), and 11 with many points (at least two) of comparable amount of fragmentation (class MF). 29 records had too low accuracy (class LA) to decide among these classes. Thus we have a new tool for classification of such meteoroids for which we have very precise data available, i.e. classification according to their ability to withstand fragmentation and their ability to then be broken suddenly in the atmosphere at a distinct height. The classification thus becomes two dimensional in separating at least partly the influence of composition from the influence of the structure of the body.

\section{1. STRENGTH CATEGORIES}

The dynamic pressure $p=\rho v^{2}$ was computed at the fragmentation points of the $1 \mathrm{~F}$ and MF cases : see histogram in Fig. 4. The precision of the individual values is better than the size of the histogram bins. Preference for some pressure values seems suggested. Thus we can define several strength categories of fireball meteoroids and determine average values of pressures at which meteoroid gross-fragmentation takes place, as proposed in Table II.

We also studied the maximum dynamic pressure acting on the NF fireballs. These pressures were mostly below $12 \mathrm{Mdyn} / \mathrm{cm}^{2}$ with 4 exceptions of very strong bodies, i.e. PN 38737, PN 39521, PN 39775B, which survived as a single body up 


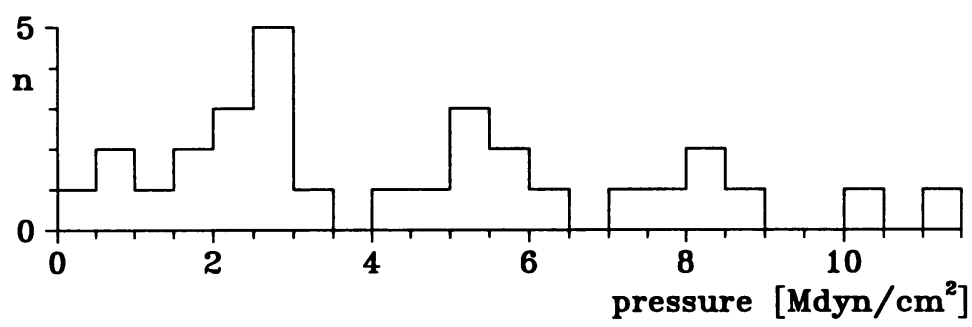

Fig. 4. Histogram of the dynamic pressures at the fragmentation points of $1 F$ and $M F$ fireballs.

TABLE II

Definition of strength categories.

\begin{tabular}{ccc}
\hline $\begin{array}{c}\text { strength } \\
\text { category }\end{array}$ & $\begin{array}{c}\text { interval of } \boldsymbol{p} \\
{\left[\mathrm{Mdyn} / \mathrm{cm}^{2}\right]}\end{array}$ & $\begin{array}{c}\text { average } \boldsymbol{p} \\
{\left[\mathrm{Mdyn} / \mathrm{cm}^{2}\right]}\end{array}$ \\
\hline & & \\
$a$ & $p<1.4$ & 0.8 \\
$b$ & $1.4 \leq p<3.9$ & 2.5 \\
$c$ & $3.9 \leq p<6.7$ & 5.3 \\
$d$ & $6.7 \leq p<9.7$ & 8.0 \\
$e$ & $9.7 \leq p<12$. & 11. \\
\hline
\end{tabular}

to $15 \mathrm{Mdyn} / \mathrm{cm}^{2}$, and $\mathrm{PN} 39935 \mathrm{~A}$, which survived up to $50 \mathrm{Mdyn} / \mathrm{cm}^{2}$.

\section{2. ABLATION}

One of the parameters determined by the model is the ablation coefficient, $\sigma$. One value of $\sigma$, which was determined for all 80 studied fireballs, is closer to the value after the fragmentation point, denoted $\sigma_{2}$. Histograms of the ablation coefficients determined for different fragmentation styles are given in Fig. 5. The ablation coefficients for the $1 \mathrm{~F}$ and MF fireballs sorted into individual groups (I, II, IIIA and IIIB) proved to be smaller than the previously published statistical values (Ceplecha, 1988), but quite close to the values derived for NF fireballs. But when the $1 \mathrm{~F}+\mathrm{MF}$ fireballs were computed as $\mathrm{NF}$ cases (i.e. as single body neglecting their fragmentation), the values of the ablation coefficients were larger and closer to their previous statistical values. This is one more justification for the validity of this fragmentation model. The resulting average values of the ablation coefficients $\sigma$ are given in Table III for different groups and different fragmentation styles. The statistics are quite small, since we chose only the most precise data available on fireballs. On the other hand, individual values within these statistics are rather precise values disclosing properties of individual meteoroids.

Table III also reveals that the gross-fragmentation is more frequent for stronger 

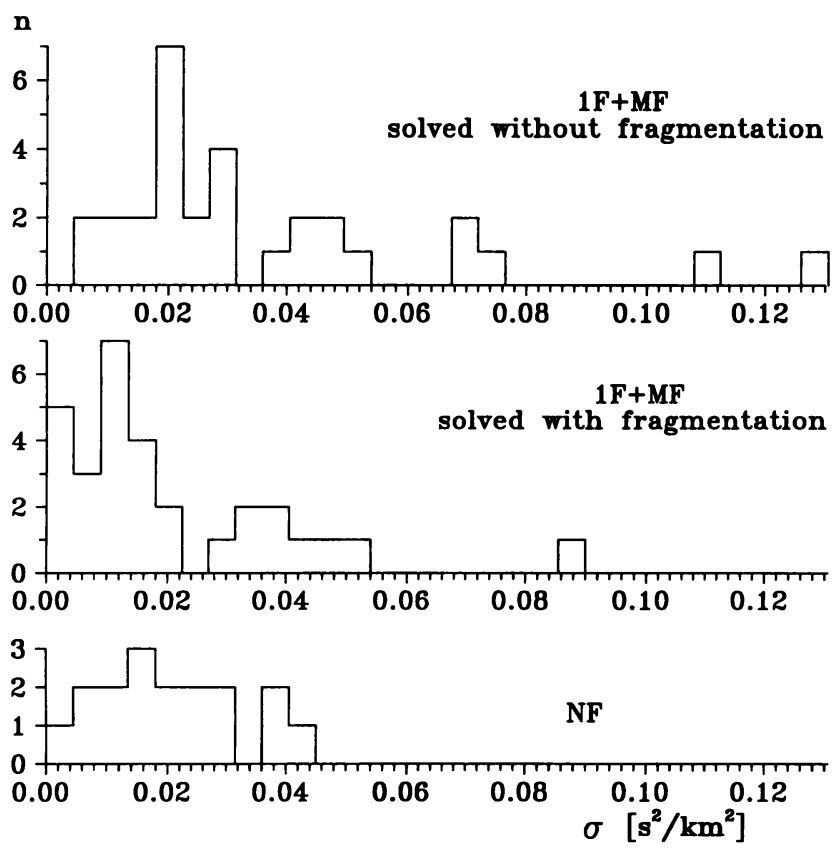

Fig. 5. Histograms of ablation coefficients show the difference between neglecting the gross fragmentation (top : higher values) and taking it into account in the fragmentation model (middle). Genuine NF cases as ascertained by the fragmentation model (bottom) show distribution similar to the $1 F$ and MF cases (middle).

\section{TABLE III}

Average ablation coefficient $\sigma$ in $\mathrm{s}^{2} / \mathrm{km}^{2}$ for different fireball groups (types). $\mathrm{n}=$ number of cases, PSV = previous statistical values (Ceplecha, 1988).

\begin{tabular}{ccccccc}
\hline type & $n$ & $\begin{array}{c}\text { 1F+MF } \\
\text { assumed } \\
\text { as NF }\end{array}$ & $1 \mathrm{~F}+\mathrm{MF}$ & $\mathrm{n}$ & $\mathrm{NF}$ & PSV \\
\hline & & & & & & \\
I & 21 & 0.023 & $\mathbf{0 . 0 1 1}$ & 12 & 0.015 & 0.017 \\
II & 8 & 0.060 & $\mathbf{0 . 0 3 8}$ & 6 & 0.035 & 0.041 \\
IIIA & 1 & 0.128 & 0.088 & 2 & 0.15 & 0.10 \\
IIIB & & & & 1 & 0.59 & 0.21 \\
& & & & & & \\
\hline
\end{tabular}

types of fireballs. For group I, there are almost twice as many $1 F+M F$ as NF whereas for group II, these two categories occur almost equally. And the only IIIB case we were able to compute with the fragmentation model is NF. But one should 
have in mind that the ablation in both the single-body and the fragmentation model contains continuous fragmentation as well. Thus the IIIB type (PN 39973) ablated only in form of continuous fragmentation without any sudden gross-fragmentation. The maximum pressure it suffered was only $0.088 \mathrm{Mdyn} / \mathrm{cm}^{2}$ at the terminal height of $75 \mathrm{~km}$ with a terminal velocity of $15.5 \pm .3 \mathrm{~km} / \mathrm{s}$ and with an ablation coefficient of $0.59 \pm .02 \mathrm{~s}^{2} / \mathrm{km}^{2}$; its initial velocity was computed as $16.555 \pm .008 \mathrm{~km} / \mathrm{s}$ and the bulk density as $0.4 \mathrm{~g} / \mathrm{cm}^{3}$. Other extreme of NF is PN 39935A, a type I fireball, which survived pressure of $50 \mathrm{Mdyn} / \mathrm{cm}^{2}$ without fragmentation. Its very low ablation coefficient $0.0010 \pm 0.0005$ guarantees that continuous fragmentation was almost completely absent.

The two dimensional classification of fireballs is also evident from Fig. 6, where the ablation coefficient $\sigma$ is plotted against the dynamic pressure at the fragmentation point. Horizontal lines divide the groups, vertical lines the strength categories. All computed $1 \mathrm{~F}$ and MF fireballs are presented : the MF cases are all in the group I region, but covering the whole $b$ to $e$ category. Thus the multiple fragmentation is typical for the type I fireballs with $p>2 \mathrm{Mdyn} / \mathrm{cm}^{2}$. About one half of the type I gross-fragmentation is realized at several points for one fireball. Type II fireballs with gross-fragmentation have usually just one fragmentation point. The numbers of cases in different fragmentation styles and for different types of fireballs are given in Table IV. Using the fragmentation model solutions, individual $\sigma$ values separate type I fireballs better from type II fireballs than using statistical criteria or individual no-fragmentation solutions.

TABLE IV

Number of cases according to fragmentation styles and fireball types.

\begin{tabular}{cccc}
\hline & & & \\
type & NF & $1 F$ & MF \\
\hline I & 12 & 11 & 10 \\
II & 6 & 7 & 1 \\
IIIA & 2 & 1 & 0 \\
IIIB & 1 & 0 & 0 \\
\hline
\end{tabular}

\section{3. BULK DENSITY}

Bulk densities can be also computed for each fireball individually providing we have a photometrically well determined mass. Fewer fireballs have had such data determined and only quite rough statements can be made. All values of bulk densities computed without fragmentation for $1 \mathrm{~F}+\mathrm{MF}$ cases are significantly lower than when computed with the fragmentation model (for the Geminids : see also Ceplecha and McCrosky, 1992). On the other hand, the bulk density values computed for all $17(1 \mathrm{~F}+\mathrm{MF})$ fireballs with relevant data by using the fragmentation model came close to the statistical values of bulk densities (Ceplecha, 1988). This revision of 


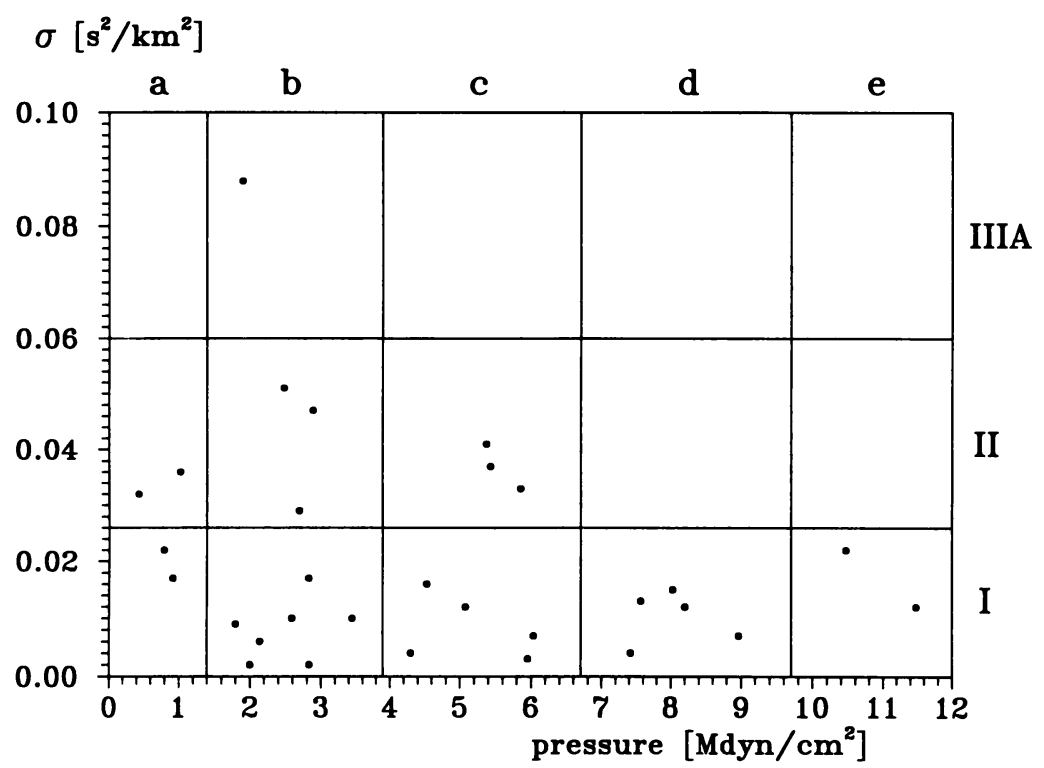

Fig. 6. Ablation coefficient $\sigma$ plotted against pressure at the fragmentation point. Each point belongs to one fireball. The grid lines are the dividing lines among different types and categories of fragmenting fireballs (ablation classes or types I, II, IIIA, and strength categories $a, b, c, d, e)$.

the average ablation coefficients and bulk densities for individual fireball groups does not change the values of the average statistical densities for groups I and II, but it makes the ablation coefficients smaller. The reason for this may be the statistical method used for the separation of $\sigma$ from $K$, because only the product $\sigma K$ was determined from observations by Ceplecha and McCrosky (1976). Thus the statistical values of bulk densities for group I is $\rho=3.7 \mathrm{~g} / \mathrm{cm}^{3}$ and for group II is $\rho=2.0 \mathrm{~g} / \mathrm{cm}^{3}$, and these seem to be close to reality. The one IIIA class fireball for which we were able to compute the bulk density gave $0.75 \mathrm{~g} / \mathrm{cm}^{3}$, identical to the statistical value. The one of IIIB classification gave $0.4 \mathrm{~g} / \mathrm{cm}^{3}$, higher than the statistical value, but probably a preferable value, because its photometry was precisely calibrated.

\section{4. FRAGMENTED MASS}

Another parameter also determined in the fragmentation model is the amount of fragmented mass, $m_{f}$, relatively to the mass immediately before gross-fragmentation. A histogram is given in Fig. 7. The amount of fragmentation for $1 \mathrm{~F}$ class is typically $60 \%$, which corresponds to breaking the body into approximately two halves with some accompanying small fragments. The second most common value is between $95 \%$ and $99 \%$, which corresponds to almost complete disruption of the body. The distribution of $m_{f}$ for the MF class is more random than for the $1 \mathrm{~F}$ class. A typical sudden fragmentation for almost half of all fragmented meteoroids 
is equivalent to stripping away slightly more than half of the mass.
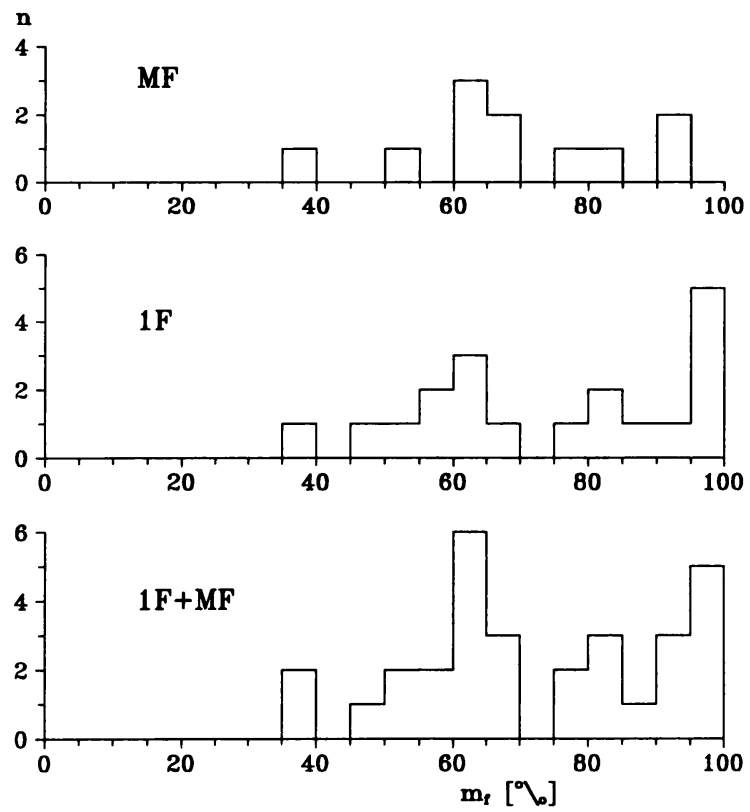

Fig. 7. Histograms of percent of fragmented mass at fragmentation points for $1 \mathrm{~F}$ and MF fireballs.

The position of the fragmentation point on the fireball trajectory is dependent on velocity. At velocities below $15 \mathrm{~km} / \mathrm{s}$, the fragmentation point usually occurs during the last time second of the trajectory. For velocities over $20 \mathrm{~km} / \mathrm{s}$, the fragmentation takes place during the first time second of the luminous trajectory. For higher velocities, gross-fragmentation might even cause the onset of luminosity, but there is not much change of velocity at the beginning point for such large bodies to decide, if this is the case (see for comparison Geminids with $v \approx 36 \mathrm{~km} / \mathrm{s}$ in Ceplecha and McCrosky, 1992). Application of the fragmentation model to fainter meteors (small masses) with enough change of velocity shortly after the beginning of the luminous trajectory may be another possibility for understanding how the beginning of a luminous trajectory is related to sudden fragmentation.

\section{Precision from independent records}

The precision of results in applying the fragmentation model may be demonstrated by independent results on the same fireball from two independent records taken at two different stations. 4 fireballs with records of good precision of dynamic data from both stations were computed. The fragmentation model was thus applied independently to two records of the same fireball and comparison of the results gave excellent agreement in all 4 cases (Table V). 


\section{TABLE V}

Fragmentation model applied to 4 cases of the same fireball with independent records from two different stations. $v_{\infty}=$ initial no-atmosphere velocity; $\sigma=$ ablation coefficient; $m_{\infty}=$ initial mass; $m_{E}=$ terminal mass; $\varepsilon_{l}=$ standard deviation for one case of observed distance along the trajectory.

\begin{tabular}{|c|c|c|c|c|c|c|}
\hline $\begin{array}{c}\text { fireball } \\
\text { no. + st. }\end{array}$ & class & $\begin{array}{c}v_{\infty} \\
{[\mathbf{k m} / \mathbf{s}]}\end{array}$ & $\begin{array}{c}\sigma \\
{\left[\mathrm{s}^{2} / \mathrm{km}^{2}\right]}\end{array}$ & $\begin{array}{l}m_{\infty} \\
{[\mathbf{k g}]}\end{array}$ & $\begin{array}{l}m_{E} \\
{[\mathbf{k g}]}\end{array}$ & $\begin{array}{c}\varepsilon_{l} \\
{[\mathrm{~m}]}\end{array}$ \\
\hline $3885615 \mathrm{E}$ & NF & $\begin{array}{r}19.757 \\
\pm .006\end{array}$ & $\begin{array}{l}0.0254 \\
\pm .0005\end{array}$ & 0.036 & 0.0009 & \pm 16 \\
\hline $3885616 \mathrm{~W}$ & NF & $\begin{array}{r}19.751 \\
\pm .011\end{array}$ & $\begin{array}{l}0.0248 \\
\pm .0006\end{array}$ & 0.033 & 0.0007 & \pm 21 \\
\hline $39055 \quad 8 \mathrm{~S}$ & $1 F$ & $\begin{array}{r}16.013 \\
\pm .003\end{array}$ & $\begin{array}{l}0.0469 \\
\pm .0005\end{array}$ & 0.78 & 0.004 & \pm 14 \\
\hline 39055 14E & $1 F$ & $\begin{array}{r}16.017 \\
\pm .002\end{array}$ & $\begin{array}{c}0.0457 \\
\pm .0002\end{array}$ & 0.75 & 0.005 & \pm 9 \\
\hline $3906516 \mathrm{E}$ & NF & $\begin{array}{r}17.321 \\
\pm .005\end{array}$ & $\begin{array}{l}0.0303 \\
\pm .0005\end{array}$ & 1.3 & 0.037 & \pm 16 \\
\hline $39065 \quad 7 \mathrm{~S}$ & NF & $\begin{array}{r}17.343 \\
\pm .010\end{array}$ & $\begin{array}{l}0.030 \\
\pm .002\end{array}$ & 1.2 & 0.12 & \pm 20 \\
\hline $3982014 \mathrm{~S}$ & MF & $\begin{array}{r}24.624 \\
\pm .002\end{array}$ & $\begin{array}{l}0.0147 \\
\pm .0006\end{array}$ & 2.7 & 0.03 & \pm 26 \\
\hline $3982015 \mathrm{~W}$ & $1 F$ & $\begin{array}{r}24.644 \\
\pm .003\end{array}$ & $\begin{array}{r}0.0129 \\
\pm .0005\end{array}$ & 2.0 & 0.02 & \pm 23 \\
\hline
\end{tabular}

Fireball PN 39820 is just a borderline case in the fragmentation classification, but it is more likely $1 \mathrm{~F}$ class, because the more precise record from station $15 \mathrm{~W}$ classifies it as 1F. This fireball actually demonstrates differences of results to be expected for borderline cases with uncertain classification between $1 F$ and MF. At this occasion it is useful to stress that also the NF class cases, when classified as NF cases by using our gross-fragmentation model, demonstrate the precision of the model. If $1 F$ or MF cases are dealt with by the single body model for the same fireball and the results for two independent records compared, the differences are 
significantly greater than those in Table V.

\section{Proposal}

Generally speaking, with the precision of the photographic fireball data (PN, EN, MORP), only the best records can be used for a detailed study of the grossfragmentation dynamics. For most of these cases, only the model with one-fragmentation point gave reasonable results. This invokes a proposal for putting into operation some system of cameras with much longer focal distances and sufficiently large fields of view. Precision in observed distances along the trajectory should be of the order of several meters. The focal length of the cameras should be around $1 \mathrm{~m}$ with large fields of view. But the biggest problem could be the realization of time marks with sufficiently high precision for fireballs photographed with such cameras.

Very recently, Hills and Goda (1993) presented a new elaborate model of meteoroid (asteroid) fragmentation during the atmospheric penetration. Even if bodies larger than the meteoroids accessible to precise photographic records are the primary concern of this paper, the smallest sizes covered by the theoretical model of Hills and Goda correspond to the largest sizes of the recorded meteoroids. Thus, some predictions of this new fragmentation theory of very large bodies could be partly checked on by photographic observations of fireballs (Prairie Network data).

\section{Acknowledgements}

My sincere thanks are due to Dr. R.E. McCrosky for his excellent Prairie Network photographs of fireball splittings and for his kindness in lending me such films from the Smithsonian Astrophysical Observatory archive located at the Oak Ridge Observatory, Harvard, MA, USA. The tedious measurements of these films with fireball splittings were realized at the Ondrejov Observatory of the Academy of Sciences of the Czech Republic by J. Keclíková using a Zeiss Ascorecord, for which work I want to express my special thanks. My thanks are due to C.-Y. Shao for his help with searching in the PN archive and for his enormous work connected with the original measurements of the PN films. I am very much indebted to Dr. J. Borovička and Dr. P. Spurný for their fragmentation computations and for very valuable scientific discussions.

This work has been supported by grant 30319 from the Czechoslovak Academy of Sciences.

\section{References}

Ceplecha, Z. : 1967, "Classification of meteor orbits." Smithson. Contr. Astrophys., 11, 35.

Ceplecha, Z. : 1968, "Discrete levels of meteor beginning height." Smithson. Astrophys. Obs.Spec. Rep., $279,1$.

Ceplecha, Z. : 1988, "Earth's influx of different populations of sporadic meteoroids from photographic and television data." Bull. Astron. Inst. Czechosl., 39, 221. 
Ceplecha, Z. : 1992, "Influx of interplanetary bodies onto Earth." Astron. Astrophys., 263, 361.

Ceplecha, Z. and Borovička, J. : 1992, "Meteors." In Interrelations between Physics and Dynamics for Minor Bodies in the Solar System (D. Benest, Cl. Froeschlé, Eds.), 309368, Editions Frontières, Gif-sur-Yvette, France.

Ceplecha, Z. and McCrosky, R.E. : 1976, "Fireball end heights : a diagnostic for the structure of meteoric material." J. Geophys. Res., 81, 6257.

Ceplecha, Z. and McCrosky, R.E. : 1992, "Gross-fragmentation of meteoroids and bulk density of Geminids from photographic fireball records." In Asteroids, Comets, Meteors 1991 (A.W. Harris and E. Bowell, Eds.), 109-112, Lunar and Planetary Institute, Houston, USA.

Ceplecha, Z., Spurný, P., Borovička, J. and Keclíková, J. : 1993, "Atmospheric fragmentation of meteoroids." Astron. Astrophys., 279, 615.

Halliday, I., Blackwell, A.T. and Griffin, A.A. : 1984, "The frequency of meteorite falls on Earth." Science, 223, 1405.

Halliday, I., Blackwell, A.T. and Griffin, A.A. : 1989, "Detailed records of many unrecovered meteorites in western Canada for which further searches are recommended." $J$. Roy. Astron. Soc. Canada, 83, 49.

Hills, J.G. and Goda, M.P. : 1993, "The fragmentation of small asteroids in the atmosphere." Astron. J., 105, 1114.

McCrosky, R.E. and Ceplecha, Z. : 1969, "Photographic networks for fireballs." In Meteorite Research (P.E. Millman, Ed.), 600, Reidel, Dordrecht.

McCrosky, R. E., Posen, A., Schwartz, G. and Shao, C.-Y. : 1971, "Lost City meteorite : its recovery and a comparison with other fireballs." J. Geophys. Res., 76, 4090.

McCrosky, R. E., Shao, C.-Y. and Posen, A. : 1977, "Prairie Network Fireball Data." Center for Astrophysics, Preprint Series, Nos. 665, 721.

Pecina, P. and Ceplecha, Z. : 1983, "New aspects in single-body meteor physics." Bull. Astron. Inst. Czechosl., 34, 102.

Pecina, P. and Ceplecha, Z. : 1984, "Importance of atmospheric models for interpretation of photographic fireball data." Bull. Astron. Inst. Czechosl., 35, 120.

Rabinowitz, D. : 1993, "The size distribution of the Earth-approaching asteroids". Astroph. J., 407, 412.

Sekanina, Z. : 1983, "The Tunguska event : no cometary signature in evidence". Astron. J., 88, 1382.

Wetherill, G.W. and ReVelle, D.O. : 1981a, "Which fireballs are meteorites ? A study of the Prairie Network Photographic Meteor Data". Icarus, 48, 308.

Wetherill, G.W. and ReVelle, D.O. : 1981b, "Relationships between comets, large meteors, and meteorites". In Comets (L. Wilkening, Ed.), 297, Univ. of Arizona Press, Tucson, USA.

\section{Late note on Strength Categories (2.1.)}

The "preference for some pressure values" was questioned by one of the referees asking for more statistics in this respect. The pattern in Fig. 4 is not random : at least it shows a systematic decrease with the increasing pressure. The fact that the highest pressure is $11.5 \mathrm{Mdyn} / \mathrm{cm}^{2} \mathrm{has}$ also a statistical significance. If the histogram is constructed up to, say, $100 \mathrm{Mdyn} / \mathrm{cm}^{2}$, the distribution is clearly not random. From the fact that the histogram is plotted from 0 to 11.5 $\mathrm{Mdyn} / \mathrm{cm}^{2}$ one cannot say it should be random inside this interval.

The observational precision of individual values of pressure is better than the chosen pressure interval of the histogram : statistical spread in such a case corresponds to the natural distribution of the events.

We should not handle each pressure interval of the histogram as an isolated case : it is the change of the numbers of cases from one interval to the neighboring interval, which makes the systematic part of the distribution. Not only the maxima, but also the minima of the distribution 
are important. The assumption that the pattern is completely random inside the plotted interval yields the average number of cases in each box $1.3 \pm 1.3$ (the standard deviation), while assumption of a linear change of number of cases with the increasing pressure yields \pm 0.7 with the $b, c, d$ maxima being significant (over one standard deviation). The distribution is not known a priori : a comparison of maxima and minima significance in Fig 4 is given in Table VI for several assumed distributions. There is no doubt about significance of the $b, c$ maxima and $b / c$ and $c / d$ minima. The $d$ maximum and $a / b$ and $d / e$ minima are significant only if the decrease of the number of cases with the increasing pressure is accounted for. The a region is defined in Table II only as all cases lower than $1.4 \mathrm{Mdyn} / \mathrm{cm}^{2}$ : there are 4 cases in this region; this is significantly more than what could be expected from a possible admixture of the $b$ distribution. The $e$ region is formed by the two largest pressure values observed. The significance of the $e$ region is clear just from the fact that there are no larger values : these two values could hardly be a statistical wing of the $d$ distribution.

If interpolation polynomial solutions are used for the whole distribution pattern in Fig. 4, then the systematic part of errors using a first order polynomial is $82 \%$; using a second order polynomial the systematic part of errors is $80 \%$; a polynomial of the order 9 yields a solution with $65 \%$ of systematic errors still left. The big change occurs first, when the order of the polynomial is chosen as 10 : the systematic error decreases to $25 \%$ only, and this polynomial yields maxima at $0.5,2.4$, $5.5,8.4,10.5 \mathrm{Mdyn} / \mathrm{cm}^{2}$, and minima at $1.2,4.0,7.0,9.9$, quite close to the values declared in Table II.

Summarizing : the $b$ maximum in Fig. 4 is the only certain. The $c$ and $d$ maxima are also over one standard deviation, but to a lesser extent than $b$ : their reality should be checked on in the future, when more data of very high precision - necessary for this analysis - will be available. The $a$ and $e$ regions are included, because of low probability that they belong to the neighboring $b$ or $d$ distributions, respectively. The extreme possibility of a single Gaussian distribution over the whole interval is in this paper superseded by the other extreme possibility that there are 5 independent Gaussian distributions inside this interval.

The following Table VI contains deviations of the maxima and the minima of Table II from the corresponding average values. These deviations are expressed in units of one standard deviation. The maxima and minima with values $\geq 1.0$ are statistically significant. The values of standard deviations are : \pm 1.3 cases in a box for the random distribution; \pm 1.1 cases in a box for the single Gaussian distribution; \pm 0.7 cases in a box for the linear distribution; \pm 0.8 cases in a box for the quadratic and the power 4 distribution; \pm 0.5 cases in a box for the quintuplet Gaussian distribution used in this paper.

\section{TABLE VI}

\begin{tabular}{c|cc|ccc}
\hline \multirow{2}{*}{$\begin{array}{c}\text { notation of } \\
\text { maximum } \\
\text { or minimum }\end{array}$} & \multicolumn{5}{c}{$\mathrm{d} \mathrm{i} \mathrm{s} \mathrm{t} \mathrm{i} \mathrm{b} \mathrm{t}$ i o $\mathrm{n}$} \\
\cline { 2 - 6 } & random & $\begin{array}{c}\text { single } \\
\text { Gaussian }\end{array}$ & \multicolumn{3}{c}{ p o l y n o m i a l } \\
linear & quadratic & power 4 \\
\hline a & 0.5 & 1.5 & - & - & - \\
a/b & 0.2 & 0.5 & 1.4 & 1.2 & 1.3 \\
b & 2.8 & 3.5 & 4.8 & 4.3 & 3.7 \\
b/c & 1.0 & 1.5 & 2.4 & 2.3 & 2.6 \\
c & 1.3 & 0.9 & 2.4 & 1.9 & 2.1 \\
c/d & 1.0 & 1.6 & 1.8 & 1.7 & 1.4 \\
d & 0.5 & 0.8 & 1.6 & 1.3 & 1.6 \\
d/e & 1.0 & 0.5 & 1.1 & 1.0 & 0.9 \\
e & 0.2 & 0.8 & 0.6 & 0.8 & 0.5 \\
\hline
\end{tabular}

